\title{
CONCERNING ARCWISE CONNECTEDNESS AND THE EXISTENCE OF SIMPLE CLOSED CURVES IN PLANE CONTINUA
}

\author{
BY \\ CHARLES L. HAGOPIAN( $\left.{ }^{1}\right)$
}

1. Introduction. If a compact metric continuum is locally connected, then it is arcwise connected. However, a semi-locally-connected compact metric continuum, even when lying in the plane, may fail to be arcwise connected [8]. A continuum $M$ is said to be aposyndetic at a point $p$ of $M$ with respect to a set $N$ in $M-\{p\}$ if there exist an open set $U$ and a continuum $H$ in $M$ such that $p \in U \subset H \subset M-N$. A continuum $M$ is said to be aposyndetic at a point $p$ if for each point $q$ in $M-\{p\}, M$ is aposyndetic at $p$ with respect to $q$. If $M$ is aposyndetic at each of its points, then $M$ is said to be aposyndetic. A compact continuum is aposyndetic if and only if it is semi-locally-connected [2]. A plane continuum $M$ is connected im kleinen at a point $x$ of $M$ if and only if for each pair of points $y$ and $z$ in $M-\{x\}, M$ is aposyndetic at $x$ with respect to $\{y, z\}$ [4].

In this paper arcwise connectedness is established for certain nonconnected im kleinen aposyndetic compact plane continua. If an aposyndetic compact plane continuum $M$ contains a finite set of points $F$ such that for each point $x$ in $M-F$, there exist two points $y$ and $z$ in $F$ such that $M$ is not aposyndetic at $x$ with respect to $\{y, z\}$, then $M$ is arcwise connected. It is also proved that each point of $M$ is in a simple closed curve which is contained in $M$ and if the set $F$ consists of two points, then $M$ is cyclicly connected (that is, for any points $a$ and $b$ in $M$, there is a simple closed curve in $M$ which contains $\{a, b\})$. Arcwise connectedness is also established for certain nonaposyndetic plane continua. If a compact plane continuum $M$ is semi-locally-connected at all except a finite number of its points and is such that for each point $x$ in $M, M$ is either not aposyndetic or not semi-locallyconnected at $x$, then $M$ is arcwise connected. For a point $x$ of a continuum $M$, F. B. Jones defines $K_{x}$ to be the closed (not necessarily connected) set consisting of $x$ and all points $y$ in $M-\{x\}$ such that $M$ is not aposyndetic at $x$ with respect to $y$ [3, Theorem 2]. Here it is proved that if $M$ is a compact plane continuum such that for each point $x$ of $M$, the set $K_{x}$ is finite, and $M$ is either not semi-locallyconnected or not aposyndetic at $x$, then each point of $M$ is in a simple closed curve which is contained in $M$. Let $M$ be a compact plane continuum which contains a point $y$ such that for each point $x$ in $M-\{y\}, M$ is semi-locally-connected at $x$ and

Received by the editors March 17, 1969.

( $\left.{ }^{1}\right)$ Supported by the Air Force Office of Scientific Research.

Copyright (C) 1970, American Mathematical Society 
$M$ is not aposyndetic at $x$ with respect to $y$. The last result of this paper indicates that if $p$ and $q$ are distinct points of $M$ and no point of $M$ cuts $M$ weakly between $p$ and $q$, then there is a simple closed curve in $M$ which contains $\{p, q\}$. Examples are given which rule out certain extensions of these results. For definitions of unfamiliar terms and phrases see [5].

\section{Aposyndetic continua.}

DEFINITION. Let $y$ and $z$ be two distinct points of a compact metric continuum $M$. Define $L_{y z}$ to be the point set consisting of $y, z$, and all points $x$ in $M-\{y, z\}$ such that $M$ is not aposyndetic at $x$ with respect to $\{y, z\}$. If $M$ is aposyndetic and there exist distinct points $x, y$, and $z$ in $M$ such that $M$ is not aposyndetic at $x$ with respect to $\{y, z\}$, then $L_{y z}$ is a subcontinuum of $M$ [6, Theorem 3].

THEOREM 1. Suppose that $M$ is an aposyndetic compact metric continuum and $x$, $y$, and $z$ are distinct points of $M$ such that $M$ is not aposyndetic at $x$ with respect to $\{y, z\}$. Then there exist two disjoint open sets $U$ and $V$ in $M-\{x\}$ containing $y$ and $z$ respectively such that if $U^{\prime}$ is an open set in $U$ which contains $y$ and $V^{\prime}$ is an open set in $V$ which contains $z$, then the $x$-component of $L_{y z}-\left(U^{\prime} \cup V^{\prime}\right)$ meets both $\mathrm{Bd} U^{\prime}$ (the boundary of $U^{\prime}$ ) and $\mathrm{Bd} V^{\prime}$.

Proof. Since $M$ is aposyndetic at $x$, there exist continua $H$ and $K$ in $M-\{y\}$ and $M-\{z\}$ respectively such that $x$ is contained in both Int $H$ (the interior of $H$ ) and Int $K$. Define $U$ and $V$ to be disjoint open sets such that $y \in U \subset M-H$ and $z \in V \subset M-K$. Let $U^{\prime}$ be an open subset of $U$ containing $y$ and let $V^{\prime}$ be an open subset of $V$ containing $z$. Let $U_{1}, U_{2}, U_{3}, \ldots$ and $V_{1}, V_{2}, V_{3}, \ldots$ be two monotone descending sequences of circular open sets which are centered on and converge to $y$ and $z$ respectively such that $U_{1} \subset U^{\prime}$ and $V_{1} \subset V^{\prime}$. For each positive integer $n$, there exists a point $x_{n}$ in Int $H \cap$ Int $K$ such that (1) the distance from $x$ to $x_{n}$ is less than $1 / n,(2)$ the set $X_{n}$ which is the $x_{n}$-component of $M-\left(U_{n} \cup V_{n}\right)$ meets both $\operatorname{Bd} U_{n}$ and $\mathrm{Bd} V_{n}$, and does not contain the point $x$, and (3) for each positive integer $m$ less than $n, X_{m} \cap X_{n}=\varnothing$. The limit superior $X$ of $X_{1}, X_{2}, X_{3}, \ldots$ is a continuum which contains $\{x, y, z\}$.

Let $w$ be a point of $X-\{y, z\}$. Suppose that $M$ is aposyndetic at $w$ with respect to $\{y, z\}$. There exist an integer $m$ and a continuum $F$ such that (1) $w$ is in Int $F$, (2) $F$ is in $M-\left(U_{m} \cap V_{m}\right)$, and (3) $X_{m} \cap F \neq \varnothing$. It follows that for each integer $n$ greater than $m$, the set $X_{n}$ does not meet $F$. But this contradicts the fact that $w$ is in the limit superior of $X_{1}, X_{2}, X_{3}, \ldots$. Hence $M$ is not aposyndetic at any point of $X-\{y, z\}$ with respect to $\{y, z\}$.

For each positive integer $n$, define $X_{n}^{\prime}$ to be the $x_{n}$-component of $M-\left(U^{\prime} \cup V^{\prime}\right)$. Note that for each $n$, since $x_{n}$ is in Int $H \cap \operatorname{Int} K, X_{n}^{\prime}$ meets both $\mathrm{Bd} U^{\prime}$ and Bd $V^{\prime}$. Also note that for each $n, X_{n}^{\prime}$ is contained in $X_{n}$. Define $X^{\prime}$ to be the limit superior of $X_{1}^{\prime}, X_{2}^{\prime}, X_{3}^{\prime}, \ldots$ It follows that $X^{\prime}$ is a subcontinuum of $X$ which meets both $\mathrm{Bd} U^{\prime}$ and $\mathrm{Bd} V^{\prime}$. Hence the $x$-component of $L_{y z}-\left(U^{\prime} \cup V^{\prime}\right)$ meets both Bd $U^{\prime}$ and $\mathrm{Bd} V^{\prime}$. 
Definition. Let $M$ be an aposyndetic compact metric continuum and let $x, y$, and $z$ be distinct points of $M$ such that $M$ is not aposyndetic at $x$ with respect to $\{y, z\}$. There exist disjoint open sets $U$ and $V$ in $M-\{x\}$ containing $y$ and $z$ respectively such that if $U^{\prime}$ is an open subset of $U$ which contains $y$ and $V^{\prime}$ is an open subset of $V$ which contains $z$, then the $x$-component of $L_{y z}-\left(U^{\prime} \cup V^{\prime}\right)$ meets both $\operatorname{Bd} U^{\prime}$ and $\mathrm{Bd} V^{\prime}$ (Theorem 1). Let $U_{1}, U_{2}, U_{3}, \ldots$ and $V_{1}, V_{2}, V_{3}, \ldots$ be two monotone descending sequences of circular open sets in $M$ which are centered on and converge to $y$ and $z$ respectively such that $U_{1} \subset U$ and $V_{1} \subset V$. For each positive integer $n$, define $Y_{n}$ to be the $x$-component of $L_{y z}-\left(U_{n} \cup V_{n}\right)$. Define $L_{y z}^{x}$ to be the limit superior of $Y_{1}, Y_{2}, Y_{3}, \ldots$ Since for each $n$, the continuum $Y_{n}$ meets both $\operatorname{Bd} U_{n}$ and $\mathrm{Bd} V_{n}, L_{y z}^{x}$ is a continuum which contains $\{x, y, z\}$.

Throughout this paper $S$ is the set of points of a simple closed surface (that is, a 2-sphere).

THEOREM 2. If an aposyndetic continuum $M$ in $S$ contains distinct points, $x, y$, and $z$ such that $M$ is not aposyndetic at $x$ with respect to $\{y, z\}$, then the subcontinuum $L_{y z}^{x}$ (as previously defined) of $M$ is locally connected.

Proof. Let $U_{1}, U_{2}, U_{3}, \ldots$ and $V_{1}, V_{2}, V_{3}, \ldots$ be two monotone descending sequences of circular regions in $S-\{x\}$ which are centered on and converge to $y$ and $z$ respectively such that $U_{1} \cap V_{1}=\varnothing$. As in the preceding definition, for each positive integer $n$, let $Y_{n}$ be the $x$-component of $L_{y z}-\left(U_{n} \cup V_{n}\right) . L_{y z}^{x}$ is the limit superior of $Y_{1}, Y_{2}, Y_{3}, \ldots$ Suppose that $L_{y z}^{x}$ is not locally connected. It follows that $L_{y z}^{x}$ is not connected im kleinen at some point $v$ in $L_{y z}^{x}-\{x, y, z\}$. There exist two circular regions $T$ and $W$ in $S$ centered on $v$ such that

(1) $T \supset \mathrm{Cl} W(\mathrm{Cl} W$ is the closure of $W)$,

(2) $\{x, y, z\} \cap \mathrm{Cl} T=\varnothing$, and

(3) there exists a sequence of mutually exclusive continua $H_{1}, H_{2}, H_{3}, \ldots$ in $L_{y z}^{x} \cap(\mathrm{Cl} T-W)$ such that each continuum meets both $\mathrm{Bd} T$ and $\mathrm{Bd} W$ and the limit inferior of the sequence is a continuum [5, Theorem 66, p. 124]. Let $q$ be a point of the limit inferior of $H_{1}, H_{2}, H_{3}, \ldots$ which is not in $\mathrm{Bd} T \cup \mathrm{Bd} W$. Let $q_{1}, q_{2}, q_{3}, \ldots$ be a sequence of points converging to $q$ such that for each positive integer $n, q_{n} \in H_{n} \cap(T-\mathrm{Cl} W)$. Let $Q_{1}, Q_{2}, Q_{3}, \ldots$ be a sequence of mutually disjoint circular open sets in $M$ which converges to $q$ such that for each positive integer $n$, the open set $Q_{n}$ is centered on $q_{n}$ and $\mathrm{Cl} Q_{n}$ is contained in $T-\mathrm{Cl} W$. For each positive integer $n$, there exists an integer $i$ such that $Y_{i} \cap Q_{n} \neq \varnothing$. It follows that there exists a sequence of mutually exclusive continua $I_{1}, I_{2}, I_{3}, \ldots$ in $L_{y z}^{x} \cap(\mathrm{Cl} T-W)$ such that for each positive integer $n$, there exists an integer $i$ such that $I_{n} \subset Y_{i}$ and the limit inferior $I$ of $I_{1}, I_{2}, I_{3}, \ldots$ is a continuum which contains $q$ and meets $\mathrm{Bd}(\mathrm{Cl} T-W)$. There exist a point $p$ and two circular regions $R$ and $E(R \supset \mathrm{Cl} E)$ centered on $q$ in $T-W$ such that (1) the point $p$ is in $(R-\mathrm{Cl} E) \cap I$ and (2) there exists a sequence of mutually disjoint continua $F_{1}, F_{2}, F_{3}, \ldots$ such that for each positive integer $n$, there exists an integer $i$ such 
that $F_{n}$ is in $I_{i} \cap(\mathrm{Cl} R-E), F_{n}$ meets both $\mathrm{Bd} R$ and $\mathrm{Bd} E$, and the limit inferior of $F_{1}, F_{2}, F_{3}, \ldots$ is a continuum in $L_{y z}^{x}$ which contains $p$.

Assume without loss of generality that the sequence $F_{1}, F_{2}, F_{3}, \ldots$ is such that for each positive integer $n$, there exist two arc-segments $R_{n}$ and $E_{n}$ such that (1) $R_{n} \subset \mathrm{Bd} R$, (2) $E_{n} \subset \mathrm{Bd} E$, and (3) each arc-segment meets $F_{1}, F_{2}, F_{3}, \ldots$ only in $F_{2 n}$ and has one endpoint in $F_{2 n-1}$ and the other endpoint in $F_{2 n+1}$. Let $p_{1}, p_{2}, p_{3}, \ldots$ be a sequence of points converging to $p$ such that for each positive integer $n$, the point $p_{n}$ is in $F_{2 n} \cap(R-\mathrm{Cl} E)$. Let $P_{1}, P_{2}, P_{3}, \ldots$ be a sequence of circular regions in $S$ such that for each positive integer $n$, the region $P_{n}$ is centered on $p_{n}$ and $\mathrm{Cl} P_{n}$ does not meet $F_{2 n-1} \cup F_{2 n+1} \cup R_{n} \cup E_{n}$. The regions of the sequence $P_{1}, P_{2}, P_{3}, \ldots$ are mutually exclusive and converge to $p$.

There exist subsequences $U_{n_{1}}, U_{n_{2}}, U_{n_{3}}, \ldots$ and $V_{n_{1}}, V_{n_{2}}, V_{n_{3}}, \ldots$ of $U_{1}, U_{2}, U_{3}, \ldots$ and $V_{1}, V_{2}, V_{3}, \ldots$ respectively such that $\mathrm{Cl} U_{n_{1}} \cap \mathrm{Cl} R=\varnothing$, $\mathrm{Cl} V_{n_{1}} \cap \mathrm{Cl} R=\varnothing$, and for each positive integer $k$, the set $F_{2 k-1} \cup F_{2 k} \cup F_{2 k+1}$ is in the $x$-component of $M-\left(U_{n_{k}} \cup V_{n_{k}}\right)$. The component of $M-\left(U_{n_{1}} \cup V_{n_{1}}\right)$ which contains $p_{1}$ is not open relative to $M$ at $p_{1}$. Hence the boundary of $P_{1}$ contains an arc-segment $S_{1}$ whose endpoints $a_{1}$ and $b_{1}$ lie in different components of $M-\left(U_{n_{1}} \cup V_{n_{1}}\right)$ such that $M \cap S_{1}=\varnothing$. There exists a simple closed curve $C_{1}$ which separates $a_{1}$ from $b_{1}$ in $S$ and contains no point of $M-\left(U_{n_{1}} \cup V_{n_{1}}\right)$ such that $C_{1} \cap S_{1}$ is connected. In $C_{1}$ there exists an arc-segment $T_{1}$ which crosses $S_{1}$, contains no point of $M \cup \mathrm{Cl} U_{n_{1}} \cup \mathrm{Cl} V_{n_{1}}$, and has its endpoints in Bd $U_{n_{1}} \cup \operatorname{Bd} V_{n_{1}}$. The component of $M-\left(U_{n_{2}} \cup V_{n_{2}}\right)$ which contains $p_{2}$ is not open relative to $M$ at $p_{2}$. Hence the boundary of $P_{2}$ contains an arc-segment $S_{2}$ whose endpoints $a_{2}$ and $b_{2}$ lie in different components of $M-\left(U_{n_{2}} \cup V_{n_{2}}\right)$ such that $M \cap S_{2}=\varnothing$. There is a simple closed curve $C_{2}$ in $U_{n_{2}} \cup V_{n_{2}} \cup(S-M)$ which separates $a_{2}$ from $b_{2}$ in $S$ such that $C_{2} \cap S_{2}$ is connected. In $C_{2}$ there is an arcsegment $T_{2}$ which crosses $S_{2}$, contains no point of $M \cup U_{n_{2}} \cup V_{n_{2}}$, and has its endpoints in $\mathrm{Bd} U_{n_{2}} \cup \mathrm{Bd} V_{n_{2}}$. Continue this process. For each positive integer $k$, there exist two arc-segments $S_{k}$ and $T_{k}$ and a simple closed curve $C_{k}$ such that (1) $S_{k}$ has endpoints $a_{k}$ and $b_{k}$ in $M$ and is contained in $(S-M) \cap \mathrm{Bd} P_{k}$, (2) $C_{k}$ separates $a_{k}$ from $b_{k}$ and contains no point of $M-\left(U_{n_{k}} \cup V_{n_{k}}\right)$, (3) $C_{k} \cap S_{k}$ is connected, and (4) $T_{k}$ is contained in $C_{k}-\left(U_{n_{k}} \cup V_{n_{k}}\right)$, meets $S_{k}$, and has its endpoints in $\mathrm{Bd} U_{n_{k}} \cup \mathrm{Bd} V_{n_{k}}$. For each positive integer $k$, since $F_{2 k-1} \cup F_{2 k} \cup F_{2 k+1}$ is in the $x$-component of $M-\left(U_{n_{k}} \cup V_{n_{k}}\right)$, one of the two arcs $R_{k}$ or $E_{k}$ must meet points of $T_{k}$ which precede, and points of $T_{k}$ which follow $S_{k} \cap T_{k}$, with respect to the order of $T_{k}$. It follows that for each positive integer $k$, there exists an arc $A_{k}$ contained in $R_{k}$ or contained in $E_{k}$ such that $A_{k} \cup T_{k}$ contains a simple closed curve $J_{k}$ which separates $a_{k}$ from $b_{k}$ in $S$ [5, Theorem 32, p. 181]. The sequence of arcs $A_{1}, A_{2}, A_{3}, \ldots$ has a limit point $u$ in $M \cap \mathrm{Bd}(\mathrm{Cl} R-E)$. Since $M$ is aposyndetic, there exist a subcontinuum $L$ of $M$ and a region $G$ containing $u$ such that the point $p$ is in Int $L$ and $G \cap L=\varnothing$. There is a positive integer $j$ such that if $k$ is an integer greater than $j$, then $a_{k}$ and $b_{k}$ are in Int $L$. Hence for some integer $n$, 
the points $a_{n}$ and $b_{n}$ are in $L$ and the arc $A_{n}$ is contained in $G$. The simple closed curve $J_{n}$ separates $a_{n}$ from $b_{n}$ in $S$ and does not meet $L$. This contradicts the fact that $L$ is connected. It follows that $L_{y z}^{x}$ is locally connected.

THEOREM 3. If an aposyndetic compact plane continuum $M$ contains a finite set of points $F$ such that for each point $x$ in $M-F$, there exist points $y$ and $z$ in $F$ such that $M$ is not aposyndetic at $x$ with respect to $\{y, z\}$, then $M$ is arcwise connected.

Proof. Define $C$ to be the finite collection of continua $\left\{L_{y z} \mid\{y, z\} \subset F\right.$ and $L_{y z}$ is a continuum $\}$. Since $M$ is connected, $C$ is a coherent collection which covers $M$. Each element of $C$ is arcwise connected (Theorem 2). Therefore $M$ is arcwise connected.

EXAmple 1. An aposyndetic compact plane continuum $M$ which contains a countable closed set of points $F$ such that for each point $x$ in $M-F$, there exist two points $y$ and $z$ in $F$ such that $M$ is not aposyndetic at $x$ with respect to $\{y, z\}$, need not be arcwise connected. To see this consider the compact plane continuum $M$ which consists of a simple closed curve $J$ and four sequences of Cantor suspensions $A_{1}, A_{2}, A_{3}, \ldots, B_{1}, B_{2}, B_{3}, \ldots, C_{1}, C_{2}, C_{3}, \ldots$, and $D_{1}, D_{2}, D_{3}, \ldots\left({ }^{2}\right)$. The elements of these sequences are joined together at their endpoints and limit on $J$ as indicated in Figure 1.

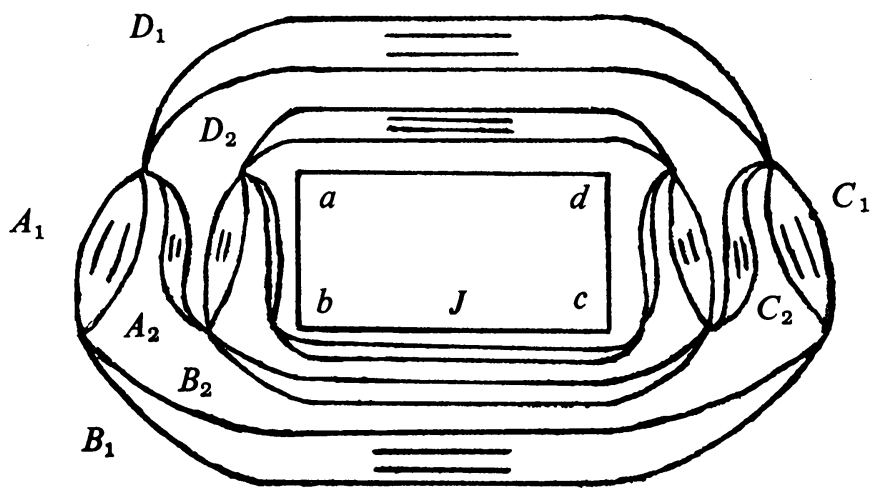

FIGURE 1

Let $a, b, c$, and $d$ denote the four points of $J$ which are limit points of the set consisting of all endpoints of Cantor suspensions in $M$. Define $F$ to be the set consisting of $a, b,{ }^{\prime} c, d$, and all endpoints of Cantor suspensions in $M . F$ is closed and countable, and for each point $x$ in $M-F$, there exist points $y$ and $z$ in $F$ such

$\left({ }^{2}\right)$ A Cantor suspension is a continuum which is the upper semicontinuous decomposition of the topological product of the unit interval $[0,1]$ and the Cantor discontinuum $C$ in which the sets $0 \times C$ and $1 \times C$ are points. These sets are referred to as endpoints of the Cantor suspension. 
that $M$ is not aposyndetic at $x$ with respect to $\{y, z\}$. Since no arc in $M$ meets both $J$ and $M-J, M$ is not arcwise connected.

EXAMPLE 2. An aposyndetic compact continuum $H$ in Euclidean 3-space which contains points $y$ and $z$ such that for each point $x$ in $H-\{y, z\}, H$ is not aposyndetic at $x$ with respect to $\{y, z\}$, may fail to be arcwise connected. Jones describes a compact plane continuum $M$ consisting of all circles centered on $\left(\frac{1}{2}, 0\right)$ and passing through a point of the Cantor discontinuum on the interval from 0 to 1 on the $x$-axis, together with alternating vertical and horizontal arcs which are each considered to be a point [4, Example 6]. $M$ is aposyndetic and not arcwise connected. Let $v$ and $w$ be distinct points of the circle of largest diameter in $M$. Let $C$ denote the Cantor discontinuum. Consider the collection of subsets of $M \times C$ which consist of the sets $v \times C$ and $w \times C$, and points of $M \times C-\{v, w\} \times C$. This collection is upper semicontinuous. With respect to its elements as points, this collection is a continuum $H$ which is aposyndetic and can be embedded in Euclidean 3-space. For each point $x$ in $H-\{y, z\} \times C, H$ is not aposyndetic at $x$ with respect to $\{y, z\} \times C$. Obviously $H$ is not arcwise connected.

THEOREM 4. Suppose that $M$ is an aposyndetic compact metric continuum and $x$, $y$, and $z$ are distinct points of $M$ such that $M$ is not aposyndetic at $x$ with respect to $\{y, z\}$. Then no point of $L_{y z}^{x}-\{x, y, z\}$ cuts $L_{y z}^{x}$ weakly between $x$ and $\{y, z\}$ (that is, for each point $p$ in $L_{y z}^{x}-\{x, y, z\}$, there is a continuum in $L_{y z}^{x}-\{p\}$ which meets both $x$ and $\{y, z\}$ ).

Proof. Assume that there exists a point $q$ in $L_{y z}^{x}-\{x, y, z\}$ which cuts $L_{y z}^{x}$ weakly between $x$ and $\{y, z\}$. Since $M$ is aposyndetic, there exists a continuum $H$ in $M-\{q\}$ which contains $x$ in its interior. Note that $H$ meets $\{y, z\}$. Now $H \cap L_{y z}$ contains no subcontinuum which meets both $x$ and $\{y, z\}$. To see this assume without loss of generality that $H \cap L_{y z}$ contains a subcontinuum $L$ which contains $x$ and $y$. The subcontinuum of $L$ irreducible from $x$ to $y$ is in $L_{y z}^{x}$ which contradicts the assumption that $q$ cuts $L_{y z}^{x}$ weakly between $x$ and $\{y, z\}$. It follows that $H \cap L_{y z}$ is the union of disjoint closed sets $A$ and $B$ containing $x$ and $\{y, z\} \cap H$ respectively. There exist disjoint open sets $U$ and $V$ which contain $A$ and $B$ respectively. $H-(U \cup V)$ is a closed subset of $M-L_{y z}$. Let $Q$ be a finite collection of continua in $M-\{y, z\}$ which cover $H-(U \cup V)$. The set $(U \cap H) \cup$ St $Q$ (St $Q$ consists of points which are contained in an element of $Q$ ) has a finite number of components each of which is closed relative to $M$. It follows that $x$ is in the interior of one of these components. Since $(U \cap H) \cup$ St $Q$ is a subset of $M-\{y, z\}$, this is a contradiction. Therefore $q$ does not cut $L_{y z}^{x}$ weakly between $x$ and $\{y, z\}$.

THEOREM 5. If an aposyndetic compact plane continuum $M$ contains points $w, x$, $y$, and $z$ such that $M$ is not aposyndetic at either $w$ or $x$ with respect to $\{y, z\}$ and $w$ is not in $L_{y z}^{x}$, then the continuum $L_{y z}^{w} \cup L_{y z}^{x}$ is cyclicly connected.

Proof. $L_{y z}^{w} \cup L_{y z}^{x}$ is locally connected (Theorem 2). Since no point of $\{y, z\}$ separates either $L_{y z}^{w}$ or $L_{y z}^{x}$ and $L_{y z}^{w} \cap L_{y z}^{x}=\{y, z\}$, no point of $\{y, z\}$ separates 
$L_{y z}^{w} \cup L_{y z}^{x}$. Suppose there is a point $q$ in $\left(L_{y z}^{w} \cup L_{y z}^{x}\right)-\{y, z\}$ which separates $L_{y z}^{w} \cup L_{y z}^{x}$. Assume without loss of generality that $q$ is in $L_{y z}^{w}$. Then $L_{y z}^{x}$ is in a component of $\left(L_{y z}^{w} \cup L_{y z}^{x}\right)-\{q\}$. It follows that $q$ cuts $L_{y z}^{w}$ between a point of $L_{y z}^{w}-\{y, z\}$ and $\{y, z\}$. This is impossible (Theorem 4). Hence $L_{y z}^{w} \cup L_{y z}^{x}$ does not contain a separating point and is cyclicly connected [7].

THEOREM 6. If an aposyndetic compact plane continuum $M$ contains points $y$ and $z$ such that for each point $x$ in $M-\{y, z\}, M$ is not aposyndetic at $x$ with respect to $\{y, z\}$, then $M$ is cyclicly connected.

Proof. For any two points $a$ and $b$ in $M$, there exist points $x$ and $w$ in $M-\{y, z\}$ such that $w$ is not contained in $L_{y z}^{x}$ and the cyclicly connected continuum $L_{y z}^{w} \cup L_{y z}^{x}$ contains $\{a, b\}$.

THEOREM 7. If an aposyndetic compact plane continuum $M$ contains a finite set of points $F$ such that for each point $x$ in $M-F$, there exist points $y$ and $z$ in $F$ such that $M$ is not aposyndetic at $x$ with respect to $\{y, z\}$, then each point of $M$ is in a simple closed curve which is contained in $M$.

Proof. For each point $x$ in $M$, define $H_{x}$ to be the finite collection

$\left\{L_{y z}^{x} \mid M\right.$ is not aposyndetic at $x$ with respect to $\{y, z\}$ and $\left.\{y, z\} \sigma F-\{x\}\right\}$.

For each point $x$ in $M$, if $H_{x}$ is nonvoid, then St $H_{x}$ is a locally connected continuum (Theorem 2) which does not contain an open subset of $M$. If $M$ is connected im kleinen at $x$, then $H_{x}$ is void. Let $p$ be a point of $M$. There exists an infinite sequence $x_{1}, x_{2}, x_{3}, \ldots$ of points of $M$ such that $x_{1}=p$ and for each positive integer $n, x_{n+1}$ is in $M-\left(F \cup \bigcup_{i=1}^{n}\right.$ St $\left.H_{x_{i}}\right)$ and the distance between $x_{n+1}$ and $p$ is less than $1 / n$. Suppose there exist a subsequence $x_{n_{1}}, x_{n_{2}}, x_{n_{3}}, \ldots$ of $x_{1}, x_{2}, x_{3}, \ldots$ and points $y$ and $z$ in $F-\{p\}$ such that for each positive integer $i$, $M$ is not aposyndetic at $x_{n_{i}}$ with respect to $\{y, z\}$. It follows that $M$ is not aposyndetic at $p$ with respect to $\{y, z\}$. Let $x(x \neq p)$ be a point of $x_{n_{1}}, x_{n_{2}}, x_{n_{3}}, \ldots$ $L_{y z}^{x} \cup L_{y z}^{p}$ is cyclicly connected (Theorem 5). Hence there exists a simple closed curve in $M$ which contains $p$.

If no such subsequence of $x_{1}, x_{2}, x_{3}, \ldots$ exists, then there exist distinct points $w$ and $x(p \notin\{w, x\})$ in $x_{1}, x_{2}, x_{3}, \ldots$ and a point $z$ in $F$ such that $M$ is not aposyndetic at either $w$ or $x$ with respect to $\{p, z\}$. It follows that the locally connected continuum $L_{p z}^{w} \cup L_{p z}^{x}$ is cyclicly connected (Theorem 5).

EXAmple 3. An aposyndetic compact plane continuum $M$ which contains a countable closed set of points $F$ such that for each point $x$ in $M-F$, there exist points $y$ and $z$ in $F$ such that $M$ is not aposyndetic at $x$ with respect to $\{y, z\}$, may contain a point which is not in a simple closed curve in $M$. To see this consider a plane continuum $M$ which consists of an arc $A$ with endpoints $a$ and $b$, and a sequence of mutually exclusive Cantor suspensions $A_{1}, A_{2}, A_{3}, \ldots$ such that (1) for each positive integer $n, a_{n}$ and $b_{n}$ are the endpoints of $A_{n}$ and $A \cap A_{n}=\left\{a_{n}, b_{n}\right\}$, 
(2) the sequences $a_{1}, a_{2}, a_{3}, \ldots$ and $b_{1}, b_{2}, b_{3}, \ldots$ converge to $a$ and $b$ respectively, and (3) $A$ is the limit set of $A_{1}, A_{2}, A_{3}, \ldots$ Let $F$ be the union of the closures of $a_{1}, a_{2}, a_{3}, \ldots$ and $b_{1}, b_{2}, b_{3}, \ldots$ For each point $x$ in $M-F$ either there exists a positive integer $n$ such that $x$ is contained in $A_{n}$ in which case $M$ is not aposyndetic at $x$ with respect to $\left\{a_{n}, b_{n}\right\}$, or $x$ is contained in $A$ and $M$ is not aposyndetic at $x$ with respect to $\{a, b\}$. No simple closed curve in $M$ meets $\{a, b\}$.

\section{Nonaposyndetic continua.}

Definition. For a point $y$ of a continuum $M$, Jones defines $L_{y}$ to be the subcontinuum of $M$ consisting of $y$ and all points $x$ in $M-\{y\}$ such that $M$ is not aposyndetic at $x$ with respect to $y$ [3, Theorem 3]. Let $x$ and $y$ be distinct points of a compact metric continuum $M$ such that $M$ is not aposyndetic at $x$ with respect to $y$. Let $V_{1}, V_{2}, V_{3}, \ldots$ be a monotone descending sequence of circular open subsets of $M\left(x \notin V_{1}\right)$ which are centered on and converge to $y$. For each positive integer $n$, let $Y_{n}$ be the $x$-component of $L_{y}-V_{n}$. Define $L_{y}^{x}$ to be the limit superior of $Y_{1}, Y_{2}, Y_{3}, \ldots$ The set $L_{y}^{x}$ is a subcontinuum of $M$ which contains $x$ and $y . M$ is not aposyndetic at any point of $L_{y}^{x}$ with respect to $y$.

Recall that for a point $z$ in $M$, the set $K_{z}$ consists of $z$ and all points $y$ of $M-\{z\}$ such that $M$ is not aposyndetic at $z$ with respect to $y$.

THEOREM 8. Suppose that $M$ is a continuum in $S$ such that for each point $z$ of $M$, the set $K_{z}$ is countable. Then if $x$ and $y$ are distinct points of $M$ such that $M$ is not aposyndetic at $x$ with respect to $y$, the subcontinuum $L_{y}^{x}$ (as previously defined) of $M$ is locally connected.

Proof. Let $V_{1}, V_{2}, V_{3}, \ldots$ be a monotone descending sequence of circular regions in $S\left(x \notin V_{1}\right)$ which are centered on and converge to the point $y$. As in the definition of $L_{y}^{x}$, for each positive integer $n$, let $Y_{n}$ be the $x$-component of $L_{y}-V_{n}$. $L_{y}^{x}$ is the limit superior of $Y_{1}, Y_{2}, Y_{3}, \ldots$ Assume that $L_{y}^{x}$ is not locally connected. It follows that $L_{y}^{x}$ is not connected im kleinen at some point $p$ in $L_{y}^{x}-\{x, y\}$. There exist two circular regions $V$ and $W$ in $S$ centered on $p$ such that (1) $V \supset \mathrm{Cl} W$, (2) $\{x, y\} \cap \mathrm{Cl} V=\varnothing$, and (3) there exists a sequence of mutually exclusive continua $H_{1}, H_{2}, H_{3}, \ldots$ in $L_{y}^{x} \cap(\mathrm{Cl} V-W)$ such that each continuum meets both $\mathrm{Bd} V$ and $\mathrm{Bd} W$ and the limit inferior of the sequence is a continuum [5, Theorem 66, p. 124]. Let $q$ be a point of the limit inferior of $H_{1}, H_{2}, H_{2}, \ldots$ which is not in $\mathrm{Bd} V \cup \operatorname{Bd} W$. Let $q_{1}, q_{2}, q_{3}, \ldots$ be a sequence of points converging to $q$ such that for each positive integer $n, q_{n} \in H_{n} \cap(V-\mathrm{Cl} W)$. Let $Q_{1}, Q_{2}, Q_{3}, \ldots$ be a sequence of mutually exclusive circular open sets in $M$ which converges to $q$ and has the property that for each positive integer $n$, the open set $Q_{n}$ is centered on $q_{n}$ and $\mathrm{Cl} Q_{n}$ is contained in $V-\mathrm{Cl} W$. For each positive integer $n$, there exists an integer $i$ such that $Y_{i} \cap Q_{n}$ $\neq \varnothing$. It follows that there exists a sequence of mutually exclusive continua $I_{1}, I_{2}, I_{3}, \ldots$ in $L_{x}^{y} \cap(\mathrm{Cl} V-W)$ such that for each positive integer $n$, there exists an integer $i$ such that $I_{n} \subset Y_{i}$ and the limit inferior $I$ of $I_{1}, I_{2}, I_{3}, \ldots$ is a continuum which contains $q$ and meets $\mathrm{Bd}(\mathrm{Cl} V-W)$. Since the set $K_{z}$ is countable 
for each point $z$ in $M$, there exist a point $z$ and two circular regions $R$ and $E$ $(\mathrm{Cl} E \subset R)$ centered on $q$ in $V-W$ such that (1) the point $z$ is in $(R-\mathrm{Cl} E) \cap I$, (2) $M$ is aposyndetic at $z$ with respect to each point of $M \cap \mathrm{Bd}(\mathrm{Cl} R-E)$, and (3) there exists a sequence of mutually exclusive continua $F_{1}, F_{2}, F_{3}, \ldots$ such that for each positive integer $n$, there exists an integer $i$ such that $F_{n}$ is in $I_{i} \cap(\mathrm{Cl} R-E)$, $F_{n}$ meets both $\mathrm{Bd} R$ and $\mathrm{Bd} E$, and the limit inferior of $F_{1}, F_{2}, F_{3}, \ldots$ is a continuum in $M$ which contains $z$.

Assume without loss of generality that the sequence $F_{1}, F_{2}, F_{3}, \ldots$ is such that for each positive integer $n$, there exist arc-segments $R_{n}$ and $E_{n}$ such that (1) $R_{n} \subset \mathrm{Bd} R$, (2) $E_{n} \subset \mathrm{Bd} E$, and (3) each arc-segment meets $F_{1}, F_{2}, F_{3}, \ldots$ only in $F_{2 n}$ and has one endpoint in $F_{2 n-1}$ and the other endpoint in $F_{2 n+1}$. Let $z_{1}, z_{2}, z_{3}, \ldots$ be a sequence of points converging to $z$ such that for each positive integer $n$, the point $z_{n}$ is in $F_{2 n} \cap(R-\mathrm{Cl} E)$. Let $Z_{1}, Z_{2}, Z_{3}, \ldots$ be a sequence of circular regions in $S$ such that for each positive integer $n$, the region $Z_{n}$ is centered on $z_{n}$ and $\mathrm{Cl} Z_{n}$ does not meet $F_{2 n-1} \cup F_{2 n+1} \cup R_{n} \cup E_{n}$. Note that the regions of the sequence $Z_{1}, Z_{2}, Z_{3}, \ldots$ are mutually exclusive and converge to $z$.

There exists a subsequence $V_{n_{1}}, V_{n_{2}}, V_{n_{3}}, \ldots$ of $V_{1}, V_{2}, V_{3}, \ldots$ such that $\mathrm{Cl} V_{n_{1}} \cap \mathrm{Cl} R=\varnothing$ and for each positive integer $k$, the set $F_{2 k-1} \cup F_{2 k} \cup F_{2 k+1}$ is in the $x$-component of $L_{y}-V_{n_{k}}$. The component of $M-V_{n_{1}}$ which contains $z_{1}$ is not open relative to $M$ at $z_{1}$. Hence the boundary of $Z_{1}$ contains an arc-segment $S_{1}$ whose endpoints $a_{1}$ and $b_{1}$ lie in different components of $M-V_{n_{1}}$ such that $M \cap S_{1}=\varnothing$. There exists a simple closed curve $C_{1}$ which separates $a_{1}$ from $b_{1}$ in $S$ and contains no point of $M-V_{n_{1}}$ such that $C_{1} \cap S_{1}$ is an arc. In $C_{1}$ there exists an arc-segment $T_{1}$ which crosses $S_{1}$, contains no point of $M \cup V_{n_{1}}$, and has its endpoints in $\mathrm{Bd} V_{n_{1}}$. The component of $M-V_{n_{2}}$ which contains $z_{2}$ is not open relative to $M$ at $z_{2}$. Hence the boundary of $Z_{2}$ contains an arc-segment $S_{2}$ whose endpoints $a_{2}$ and $b_{2}$ lie in different components of $M-V_{n_{2}}$ such that $M \cap S_{2}=\varnothing$. There is a simple closed curve $C_{2}$ which separates $a_{2}$ from $b_{2}$ in $S$ and contains no point of $M-V_{n_{2}}$ such that $C_{2} \cap S_{2}$ is an arc. In $C_{2}$ there is an arc-segment $T_{2}$ which crosses $S_{2}$, contains no point of $M \cup V_{n_{2}}$, and has its endpoints in $\operatorname{Bd} V_{n_{2}}$. Continue this process. For each positive integer $k$, there exist two arc-segments $S_{k}$ and $T_{k}$ such that (1) $S_{k}$ has endpoints $a_{k}$ and $b_{k}$ in $M$ and is contained in $(S-M) \cap \mathrm{Bd} Z_{k}$, (2) $T_{k}$ contains no point of $M \cup V_{n_{k}}$, and (3) $T_{k} \cup \operatorname{Bd} V_{n_{k}}$ contains a simple closed curve which separates $a_{k}$ from $b_{k}$ in $S$. For each positive integer $k$, since $F_{2 k-1} \cup F_{2 k} \cup F_{2 k+1}$ is in the $x$-component of $L_{y}-V_{n_{k}}$, one of the two arcs $\mathrm{Cl} R_{k}$ or $\mathrm{Cl} E_{k}$ must meet points of $T_{k}$ which precede, and points of $T_{k}$ which follow $S_{k} \cap T_{k}$, with respect to the order of $T_{k}$. It follows that for each positive integer $k$, there exists an arc $A_{k}$ contained in $R_{k}$ or contained in $E_{k}$ such that $A_{k} \cup T_{k}$ contains a simple closed curve $J_{k}$ which separates $a_{k}$ from $b_{k}$ in $S$ [5, Theorem 32, p. 181]. The sequence of arcs $A_{1}, A_{2}, A_{3}, \ldots$ has a limit point $u$ in $M \cap \mathrm{Bd}(\mathrm{Cl} R-E)$. Since $M$ is aposyndetic at $z$ with respect to each point of $M \cap \mathrm{Bd}(\mathrm{Cl} R-E)$, there exist a subcontinuum $H$ of $M$ and a region $G$ in $S$ 
containing $u$ such that the point $z$ is in the interior of $H$ and $G \cap H=\varnothing$. There exists a positive integer $j$ such that if $k$ is an integer greater than $j$, then $a_{k}$ and $b_{k}$ are in the interior of $H$. Hence for some integer $n, a_{n}$ and $b_{n}$ are in $H$ and $A_{n}$ is contained in $G$. The simple closed curve $J_{n}$ separates $a_{n}$ from $b_{n}$ in $S$ and does not meet $H$. This contradicts the fact that $H$ is a continuum. It follows that $L_{y}^{x}$ must be locally connected.

THEOREM 9. If $M$ is a compact plane continuum such that $M$ is semi-locallyconnected at all except a finite number of its points, and for each point $x$ in $M, M$ is either not aposyndetic at $x$, or not semi-locally-connected at $x$, then $M$ is arcwise connected.

Proof. If $M$ is semi-locally-connected, then $M$ is aposyndetic [2]. Hence there exists a nonvoid finite set of points $\left\{y_{1}, y_{2}, \ldots, y_{n}\right\}$ in $M$ such that (1) for each $i$ $(i=1,2, \ldots, n), M$ is not semi-locally-connected at $y_{i}$ and $L_{y_{i}}$ is arcwise connected (Theorem 8), and (2) $\bigcup_{i=1}^{n} L_{y_{i}}=M$. Since $M$ is the union of a finite number of arcwise connected continua, it is arcwise connected.

COROLlaRY. If $M$ is a totally nonaposyndetic compact plane continuum which is semi-locally-connected at all except a finite number of its points, then $M$ is arcwise connected.

EXAMPLE 4. A totally nonaposyndetic compact plane continuum which is semilocally-connected at all except a countable number of its points need not be arcwise connected. To see this consider the compact plane continuum $M$ which consists of two simple closed curves $L$ and $J$ which meet at one point $p$, and a sequence of Cantor suspensions $S_{1}, S_{2}, S_{3}, \ldots$ (each suspension having its endpoints identified) such that $S_{1} \cap L=\{p, q\}$ ( $q$ is the endpoint of $S_{1}$ ), and $S_{2}, S_{3}, S_{4}, \ldots$ are joined together and limit on $L$ and $J$ as indicated in Figure 2. $M$ is totally nonaposyndetic and is semi-locally-connected at all points except $p$, and the endpoints of the elements of $S_{1}, S_{2}, S_{3}, \ldots$. But there does not exist an arc in $M$ which has one endpoint in $S_{2}$ and the other in $L$. Note that for each point $x$ of $M$, the set $K_{x}$ consists of only two points.

The next two examples indicate that these results do not hold in Euclidean 3-space.

EXAMPLE 5. A totally nonaposyndetic compact continuum in Euclidean 3-space which is semi-locally-connected at all but a finite number of its points may fail to be arcwise connected. Let $M$ be the plane continuum described by Jones which is aposyndetic and not arcwise connected [4, Example 6]. Let $v$ be a point on the circle of largest diameter in $M$. Let $C$ denote the Cantor discontinuum. Consider the collection of subsets of $M \times C$ which consists of the set $v \times C$ and points of $M \times C-v \times C$. This collection is upper semicontinuous. With respect to its elements as points, this collection is a continuum $H$ which is semi-locally-connected 


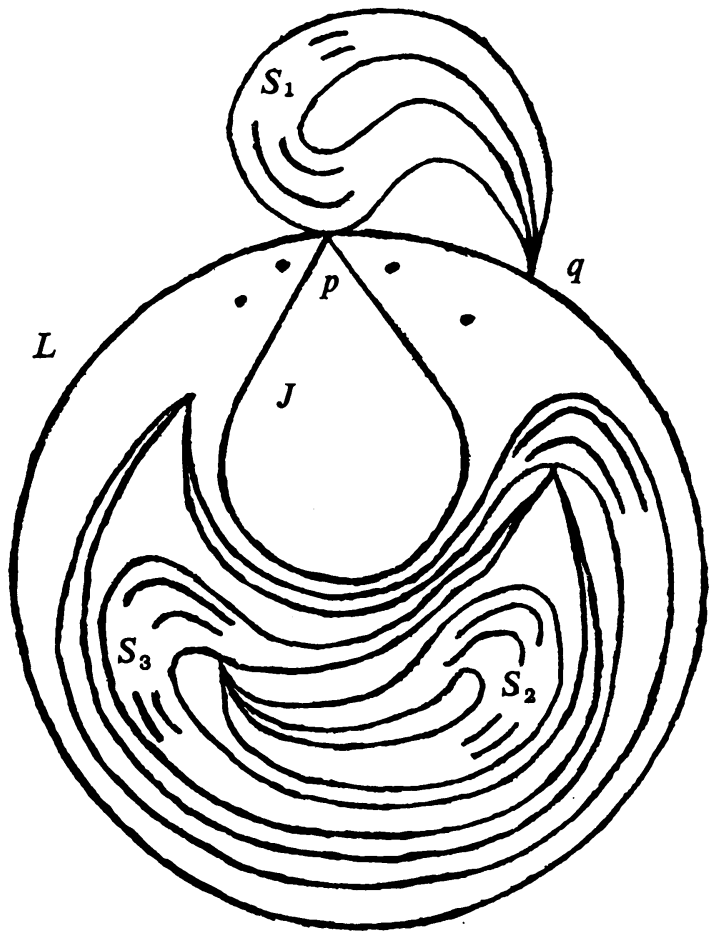

Figure 2

at each point of $H-v \times C$ and is not aposyndetic at any point of $H-v \times C$ with respect to the point $v \times C$. $H$ is not arcwise connected. But $H$ is aposyndetic at $v \times C$. However, by appropriately joining two copies of $H$, it is possible to construct a totally nonaposyndetic compact continuum in Euclidean 3-space which is semilocally-connected at all but two of its points and is not arcwise connected.

EXAMPLE 6. There exists in Euclidean 3-space a totally nonaposyndetic compact continuum which is semi-locally-connected at all but two of its points and does not contain an arc. To see this let $p$ be a point of a nondegenerate chainable hereditarily indecomposable continuum $P$ ( $P$ is the pseudo-arc) and define $G$ to be the collection of subsets of $P \times P$ consisting of the set $p \times P$ and points of $P \times P-p \times P$. $G$ is upper semicontinuous. Since $P \times P$ can be embedded in Euclidean 3-space [1], with respect to its elements as points, $G$ is a continuum $M$ in Euclidean 3-space. $M$ is semi-locally-connected at each point of $M-p \times P$ and is not aposyndetic at any point of $M-p \times P$ with respect to the point $p \times P$. Since $P$ is hereditary indecomposable, $M$ does not contain an arc. $M$ is aposyndetic at the point $p \times P$. However, by appropriately connecting two copies of this continuum, one can construct a totally nonaposyndetic continuum in Euclidean 3-space which is semi-locallyconnected at all but two of its points and does not contain an arc. 
THEOREM 10. Suppose that $x$ and $y$ are points of a compact metric continuum $M$ such that $M$ is not aposyndetic at $x$ with respect to $y$. Then if a point $q$ of $L_{y}^{x}-\{x, y\}$ cuts $M$ weakly between $x$ and $y, M$ is not aposyndetic at $x$ with respect to $q$.

Proof. Assume that a point $q$ of $L_{y}^{x}-\{x, y\}$ cuts $M$ weakly between $x$ and $y$, and $M$ is aposyndetic at $x$ with respect to $q$. There exists a continuum $H$ in $M-\{q\}$ which contains $x$ in its interior. $H \cap L_{y}$ contains no subcontinuum containing both $x$ and $y$. To see this suppose there is a subcontinuum $L$ of $H \cap L_{y}$ which contains $\{x, y\}$. Let $I$ be a subcontinuum of $L$ which is irreducible from $x$ to $y$. $I$ is contained in $L_{y}^{x}$. Therefore $q$ is in $I$. Since $H$ is in $M-\{q\}$, this is impossible. It follows that $H \cap L_{y}$ is the union of disjoint closed sets $A$ and $B$ containing $x$ and $y$ respectively. There exist disjoint open sets $U$ and $V$ which contain $A$ and $B$ respectively. $H-(U \cup V)$ is a closed subset of $M-L_{y}$. Let $Q$ be a finite collection of continua in $M-\{y\}$ which covers $H-(U \cup V)$. The set $(U \cap H) \cup \mathrm{St} Q$ has a finite number of components each of which is closed relative to $M$. It follows that $x$ is in the interior of one of these components. Since this component is contained in $M-\{y\}$, this is a contradiction. Hence $M$ is not aposyndetic at $x$ with respect to $q$.

THEOREM 11. If $M$ is a compact plane continuum such that for each point $x$ in $M$, the set $K_{x}$ is finite, and $M$ is either not semi-locally-connected at $x$ or not aposyndetic at $x$, then each point of $M$ is in a simple closed curve which is contained in $M$.

Proof. If $p$ is a point of $M$ and $M$ is not semi-locally-connected at $p$, then there exists a point $x$ in $M$ such that $M$ is not aposyndetic at $x$ with respect to $p$. Since the continuum $L_{p}^{x}$ is locally connected (Theorem 8 ) and $K_{x}$ is finite, there exists a point $q$ in $L_{p}^{x}$ such that no point of $L_{p}^{x}$ separates $p$ from $q$ in $L_{p}^{x}$ (Theorem 10). It follows that $L_{p}^{x}$ contains a simple closed curve which contains $p$ and $q$ [7]. If $M$ is semi-locally-connected at $p$, then there exists a point $y$ in $M$ such that $M$ is not aposyndetic at $p$ with respect to $y$. It follows from the same argument that in $L_{y}^{p}$ there is a simple closed curve which contains $p$.

Note that the continuum $M$ in Example 4 has all the properties stated in Theorem 11.

COROLLARY. If $M$ is a totally nonaposyndetic compact plane continuum which is semi-locally-connected at all except a finite number of its points, then each point of $M$ is in a simple closed curve which is contained in $M$.

EXAMPLE 7. If $M$ is a totally nonaposyndetic compact plane continuum such that for each point $x$ in $M$, the set $K_{x}$ is countable, then each point of $M$ need not be contained in a simple closed curve. To see this consider the plane continuum $M$ consisting of four sequences of Cantor suspensions $R_{1}, R_{2}, R_{3}, \ldots, S_{1}, S_{2}, S_{3}, \ldots$, $T_{1}, T_{2}, T_{3}, \ldots$, and $Y_{1}, Y_{2}, Y_{3}, \ldots$ (each suspension having its endpoints identified), and a point $p$ such that (1) $S_{1}, S_{2}, S_{3}, \ldots$ and $T_{1}, T_{2}, T_{3}, \ldots$ converge to $p$, (2) for each positive integer $i, R_{i}$ and $Y_{i}$ have the same endpoint, and (3) the sets 


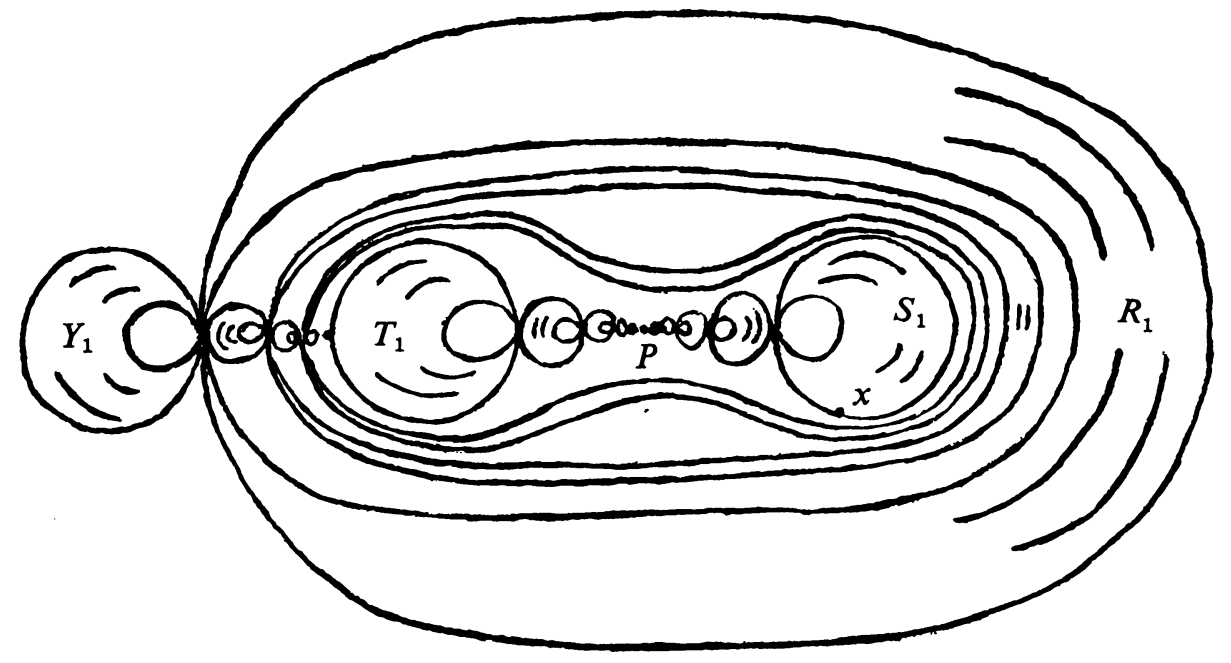

FIGURE 3

$R_{1}, R_{2}, R_{3}, \ldots$ loop around and limit on a subset of $\bigcup_{i} S_{i} \cup T_{i}$ as indicated in Figure 3. $M$ is totally nonaposyndetic. The point $p$ is not in a simple closed curve contained in $M$. Note that if $x$ is a point of $S_{1}$ which is in the limit set of $R_{1}, R_{2}, R_{3}, \ldots$ then the set $K_{x}$ is countably infinite.

THEOREM 12. Suppose that $M$ is a compact plane continuum which contains a point $y$ such that for each point $x$ in $M-\{y\}, M$ is semi-locally-connected at $x$ and $M$ is not aposyndetic at $x$ with respect to $y$. Then if $p$ and $q$ are distinct points of $M$ and no point cuts $M$ weakly between $p$ and $q$, there exists a simple closed curve in $M$ which contains $\{p, q\}$.

Proof. Assume without loss of generality that $p \neq y$. The point $q$ is in $L_{y}^{p}$ for if it were not $y$ would cut $M$ weakly between $p$ and $q . L_{y}^{p}$ is locally connected (Theorem 8 ) and does not contain a separating point (Theorem 10). Hence in $L_{y}^{p}$ there is a simple closed curve which contains $\{p, q\}$.

The following example indicates that the words "cuts $M$ weakly" in the statement of Theorem 12 cannot be replaced by "separates $M$ ".

EXAmple 8. Let $I$ be the unit interval and let $C$ be the Cantor discontinuum in $I$. For each point $r$ in $I$, let $V(r)$ be the vertical line $\{(r, y) \mid y \in I\}$ in $I \times I$. For each positive integer $n$, define $T_{n}$ to be the collection

$\{\mathrm{Cl} H \mid H$ is an open segment in $(V(1 / 3 n) \cup V(1-1 / 3 n))-I \times C$ which has both endpoints in $I \times C$ and diameter $1 / 3 n\}$.

Let $G$ be the collection consisting of $\bigcup_{n=1}^{\infty} T_{n}$, the set $V(0) \cup V(1)$, and points of $I \times C-\left(V(0) \cup V(1) \cup \bigcup_{n=1}^{\infty} \mathrm{St} T_{n}\right) . G$ is upper semicontinuous. With respect to its elements as points, $G$ is a continuum $M$. For each point $x$ in $M-(V(0) \cup V(1))$, 
$M$ is semi-locally-connected at $x$ and is not aposyndetic at $x$ with respect to the point $V(0) \cup V(1)$. $M$ does not contain a separating point and is not cyclicly connected. Note that $V(0) \cup V(1)$ is a weak cut point in $M$.

\section{BIBLIOGRAPHY}

1. R. Bennett, Embedding products of chainable continua, Proc. Amer. Math. Soc. 16 (1965), 1026-1027.

2. F. B. Jones, Aposyndetic continua and certain boundary problems, Amer. J. Math. 63 (1941), 545-553.

3. - Concerning non-aposyndetic continua, Amer. J. Math. 70 (1948), 403-413.

4. - Concerning aposyndetic and non-aposyndetic continua, Bull. Amer. Math. Soc. 58 (1952), 137-151.

5. R. L. Moore, Foundations of point-set theory, rev. ed., Amer. Math. Soc. Colloq. Publ., vol. 13, Amer. Math. Soc., Providence, R. I., 1962.

6. E. J. Vought, n-aposyndetic continua and cutting theorems, Trans. Amer. Math. Soc. 140 (1969), 127-135.

7. G. T. Whyburn, Cyclicly connected continuous curves, Proc. Nat. Acad. Sci. U.S.A. 13 (1927), 31-38.

8. — Semi-locally-connected sets, Amer. J. Math. 61 (1939), 733-749.

\section{California Institute of Technology,} Pasadena, California 